\title{
ASPECTOS GERONTOLÓGICOS DO CUIDADO CRÍTICO ÀS PESSOAS IDOSAS COM COVID-19
}

\author{
Larissa Venturini' \\ ORCID: 0000-0002-5401-3849
}

Sandra da Silva Kinalski"

ORCID: 0000-0002-4841-2288

Eliane Raquel Rieth Benetti'

ORCID: 0000-0003-1626-5698

'Universidade Federal de Santa Maria (UFSM). Santa Maria, Rio Grande do Sul, Brasil.

"Universidade Regional Integrada do Alto Uruguai e das Missões. Rio Grande do Sul, Brasil.

Autor Correspondente: Larissa Venturin E-mail: larissa.venturini@hotmail.com

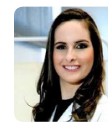

Como citar:

Venturini L, Kinalski SS, Benetti ERR. Aspectos gerontológicos do cuidado critico às pessoas idosas com covid-19. In: Santana RF. Enfermagem gerontologica no cuidado do idoso em tempos da COVID 19. 2.ed.rev. Brasilia, DF: Editora ABEn; 2020. p.55-60.

(Serie Enfermagem e Pandemias, 1). https://doi.org/10.51234/aben.20.e01.c09

\section{INTRODUÇÃO}

As doenças infecciosas emergentes e reemergentes representam um desafio para a saúde pública mundial. Nos últimos anos foram registradas várias epidemias virais, como o Coronavírus da Síndrome Respiratória Aguda Grave (SARS-CoV) em 2002 a 2003, a Influenza H1N1 em 2009, o Coronavírus da Síndrome Respiratória do Oriente Médio (MERS-CoV) em 2012. No final de 2019, foram detectados casos de SARS pelo novo Coronavírus (CoV-2) em Wuhan, na China, com posterior propagação mundial. A Organização Mundial da Saúde (OMS) nomeou, em 11 de fevereiro de 2020, a doença do Coronavírus 2019 como COVID-19 e, em 11 de março declarou a infecção por SARS-CoV-2 como uma pandemia ${ }^{(1)}$.

A COVID-19, reconhecida pela OMS como uma emergência de saúde pública internacional, tem revelado diversas facetas de saúde, patogenia, epidemiologia e cuidados. De acordo, com informações geradas no tratamento da epidemia na China, onde começou a doença, ou na Itália, país com dados alarmantes frente ao número incipiente e massivo de óbitos, tornou-se evidente que algumas pessoas têm um risco aumentado de contágio e de desenvolverem a forma grave da doença, assim necessitando internação e cuidados em Unidade de Terapia Intensiva (UTI).

Entre os mais suscetíveis, estão as pessoas idosas (independente de sua condição de saúde), pessoas com doenças crônicas, como diabetes mellitus, doenças cardiovasculares, doença pulmonar obstrutiva crônica, insuficiência renal crônica, doenças hemato-oncológicas, pacientes transplantados ou pessoas que fazem tratamento com imunossupressores. Assim, identifica-se que as pessoas idosas enfrentam mais ameaças e desafios frente à realidade posta, visto suas alterações fisiológicas decorrentes do processo de envelhecimento, que impactam diretamente em sua função imunológica, e devido possíveis condições de saúde subjacentes. 
Sabe-se também, que a população idosa, devido ao fator cronológico e patologias típicas como demência, acidente vascular cerebral, fraturas, somadas à suscetibilidade a infecções e fenômenos embólicos ${ }^{(2)}$, associado a atual infecção por COVID-19, conspiram para fazer desse grupo etário um doente crítico em potencial. Dados divulgados pelo Ministério da Saúde (MS) revelam que cerca de sete entre cada dez pessoas idosas, no Brasil, possuem pelo menos uma doença crônica. Relatos de pesquisas têm apresentado que oito em cada dez mortes por COVID-19 ocorrem em indivíduos com pelo menos uma comorbidade.

Os dados apresentados pelo $\mathrm{MS}^{(3)}$ até a data de 14 de abril, indicam que em nosso país $73 \%$ dos óbitos atribuídos à COVID-19 encontram-se na faixa etária acima de 60 anos, enquanto na Itália esses resultados atingem 95\%. As evidências vinculadas a necessidade de internação e de ocupação de leitos de UTI alinham-se à ocupação predominante pelas pessoas idosas, mesmo anterior a situação atual da pandemia, em que as admissões dessas pessoas em UTI apresentaram frequência média de 42 a 52\% (em alguns casos chegando até a $90 \%)$, consumindo cerca de $60 \%$ dos leitos disponíveis ${ }^{(3)}$. Dessa forma, corroborando ao constructo de que essa população apresenta maior risco e possui necessidades de proteção particulares, tornam-se primordiais os aprofundamentos clínicos, teóricos e reflexivos que possam impactar positivamente essa realidade.

Na atenção à saúde da pessoa idosa no cenário de cuidados intensivos, os enfermeiros podem se deparar com diferentes lacunas, muitas vezes, relacionadas aos conhecimentos e habilidades de intensivismo e de gerontogeriatria, fundamentais às boas práticas no processo de cuidar como centralidade da Enfermagem. Com o aumento da hospitalização de pessoas idosas em terapia intensiva por COVID-19, faz-se necessário que os enfermeiros de cuidados intensivos, compreendam as alterações fisiológicas associadas à senescência e as particularidades de cuidados relacionados a pessoa idosa.

Já que os processos fisiológicos são alterados em função do envelhecimento e embora essas alterações sejam progressivas, geralmente não são aparentes ou patológicas. Assim sendo, a pessoa idosa gravemente enferma requer observação mais intensa. Na perspectiva de contribuir para a expansão do cuidado à pessoa idosa com COVID-19, em situações críticas ou potencialmente críticas de cuidado, o presente estudo traz reflexões sobre a abordagem singular a esses indivíduos e as particularidades evidenciadas pelas infecções por SARS-CoV-2 em pessoas idosas.

\section{OBJETIVO}

Tecer reflexões sobre manifestações clínicas e implicações da COVID-19 nas pessoas idosas em cuidados críticos.

\section{MÉTODO}

Trata-se de texto teórico-reflexivo que versa sobre as manifestações clinicas típicas de idosos em cuidados críticos e as implicações no cuidado aos idosos com a COVID-19, assim como para a enfermagem gerontológica.

\section{RESULTADOS}

\section{MANIFESTAÇÕES CLINICAS NAS PESSOAS IDOSAS COM COVID-19 EM CUIDADOS CRÍTICOS}

O aumento da fragilidade das pessoas idosas é fator determinante para a apresentação grave da COVID-19, relacionada ao agravamento dos resultados e ao aumento da mortalidade em cuidados intensivos. Isso demanda planejamento dos cuidados para aqueles em maior risco, bem como a tomada de decisões, em conjunto com a pessoa idosa, sobre as expectativas do tratamento e sobre cuidados em fim de vida. As consequências da admissão em UTI devem ser consideradas, e os dados já nos mostram que o coronavírus afeta desproporcionalmente aqueles que são frágeis e/ou com condições crônicas de saúde ${ }^{(4)}$. Um dado 
preocupante, visto em todo o mundo diante dessa pandemia, no entanto nem todas as pessoas idosas poderão acessar o nível de atendimento de que precisam.

A COVID-19 é uma doença do trato respiratório que geralmente causa febre, tosse, fadiga, dispneia, mialgias, dor de garganta, dor de cabeça e calafrios ${ }^{(5)}$. Raramente foram identificados congestão nasal e diarreia. Queixas de dispneia ou anorexia foram mais comuns entre os pacientes admitidos em UTI $(P<0,001)^{(5)}$, sendo a falta de ar a queixa mais comum entre as pessoas idosas ( $12 \%$ para pacientes $>60$ anos e $3 \%$ para pacientes $<60$ anos) ${ }^{(6)}$.

Em alguns pacientes, especialmente em pessoas idosas, o novo coronavírus, SARS-CoV-2, e a doença associada COVID-19, podem desencadear uma apresentação sintomática de pneumonia viral bilateral grave, o que enseja a necessidade de cuidados intensivos e invasivos, como o uso de ventilação mecânica. As estatísticas mundiais têm revelado que $5 \%$ de todos os pacientes acometidos desenvolvem a apresentação grave da doença e tem indicação para tratamento em UTI, devido a Síndrome do Desconforto Respiratório Agudo (SDRA), sepse e choque séptico, repercutindo em altas taxas de mortalidade. As pessoas idosas sintomáticas são mais propensas a requerer admissão na UTI $(9,6 \%$ vs $1,4 \%, \mathrm{P}<0,001)$ e SDRA $(16,9 \%$ vs $5,4 \%, \mathrm{P}<0,001)$ em comparação com pacientes com menos de 60 anos de idade ${ }^{(6)}$.

A SDRA é um tipo de insuficiência respiratória, presente nos casos de COVID-19, que deve ser tratada conforme evidências de suas diretrizes específicas. Nos casos de acesso limitado à ventilação invasiva, pode ser utilizado oxigênio nasal de alto fluxo ou ventilação não invasiva. Entretanto, nessas duas modalidades o alto fluxo de gás é menos contido do que em circuito fechado típico dos ventiladores, o que representa risco de dispersão do vírus aerossolizado no ambiente de saúde. Problema potencial presente também, em situações mais simples, como na configuração de uma máscara facial mal ajustada. Nesse ínterim, determinar a magnitude desses riscos é um desafio e uma lacuna crucial do conhecimento ${ }^{(7)}$.

Todavia, os procedimentos de acesso invasivo das vias respiratórias e os Procedimentos Geradores de Aerossóis (PGAs), comumente realizados em terapia intensiva, devem dispor de cuidados específicos quando realizados em pacientes com a COVID-19, especialmente em pessoas idosas, diante das alterações anatômicas advindas com o envelhecimento. Por ser considerado um PGAs, para a intubação orotraqueal deve se disponibilizar o número mínimo de profissionais exigido para o procedimento, reservando os demais em outro ambiente caso seja necessário auxiliar na atuação. Também, preconiza-se o uso de Equipamentos de Proteção Individual (EPIs) recomendados pela Precaução por Aerossóis e Precaução de Contato, como máscara N95, propés, protetor facial e luvas apropriadas (mais extensas) ${ }^{(9)}$.

A rotina em terapia intensiva e os cuidados críticos dispensados às pessoas idosas que adentram esse cenário têm evidenciado singularidades vinculadas às alterações de senescência, senilidade e demais especificidades atreladas à população idosa. $\mathrm{O}$ fato de que essas pessoas possuem apresentações atípicas de algumas patologias é indiscutível no campo da gerontogeriatria, e tal evidência se apresenta e repercute frente a prevenção, manifestações clínicas, tratamento e cuidados diante da pandemia do novo coronavírus.

A apresentação diferenciada da doença em pessoas idosas é um desafio constante para a equipe multiprofissional da UTI, em especial para a enfermagem, considerando que é de responsabilidade desses profissionais garantir a observação e monitorização constante, o controle das alterações do quadro clínico e das repercussões do COVID-19, bem como reportá-las para os demais integrantes para provimento de intervenções adequadas.

No ambiente da terapia intensiva, essas alterações expõem a pessoa idosa em situação crítica e potencialmente crítica ao maior risco de complicações, de modo que é necessário que o cuidado seja focado em três pontos importantes, a saber: a prevenção de problemas potenciais, com base nas fragilidades esperadas em pessoas idosas; a diferenciação entre o que pode ser considerado fisiológico do envelhecimento e o que já é patológico, causado pela COVID-19 e precisa ser precocemente reconhecido e tratado; e o provimento de cuidados voltados para os resultados centrados na pessoa idosa com a COVID-19, considerando as singularidades apresentadas por essa população no percurso da doença ${ }^{(2)}$. 
Na rotina assistencial, pessoas idosas acometidas por vírus respiratórios apresentam algumas particularidades, que por vezes, dificultam a triagem e diagnóstico. Nesse sentido, a sintomática das pessoas idosas acometidas por COVID-19, requerem algumas ponderações. Com o envelhecimento, o sistema respiratório sofre alterações estruturais e funcionais. Como resultado da calcificação das cartilagens costais, ocorre um aumento no gasto energético despendido na respiração, com consequente utilização da musculatura acessória. A redução da complacência pulmonar e da área de superfície alveolar levam a um aumento do volume residual pulmonar e do espaço fisiológico morto, diminuindo a eficiência da troca de gases. Diante dessas alterações, o centro respiratório apresenta sensibilidade reduzida, o consumo de oxigênio diminui e a produção de gás carbônico aumenta ${ }^{(8)}$. Por isso sintomas de cansaço respiratório podem se apresentar abruptamente nos idoso, pela adaptação ruim a perda progressiva, e o exame deve se concentrar no uso da musculatura acessória respiratória.

Nesta perspectiva, nas pessoas idosas a hipoxemia por apresentar alterações no sistema respiratório ocasionado pela senescência, implicando com maior frequência de intubação endotraqueal ${ }^{(9)}$. Ademais, as pessoas idosas são mais suscetíveis do que indivíduos jovens às lesões laríngeas e a alterações vocais decorrentes desse procedimento invasivo. Isso porque, a mucosa, os músculos e cartilagens laríngeas se tornam frágeis e suscetíveis a lesões com a idade avançada, o que requer atenção em casos que exigem intubação prolongada ${ }^{(8)}$.

O novo coronavírus causa principalmente infecções pulmonares. Essas por sua vez, aumentam a sobrecarga cardíaca que, ao mesmo tempo, pode levar ao alto nível de açúcar no sangue, o que dificulta o controle da infecção. Nesse sentido, pode-se afirmar que a infecção pela COVID-19, apresenta características de uma doença multissistêmica, que coexistem nas pessoas idosas e as levam a quadros clínicos complicados e complexos.

A idade não é considerada fator de risco isolado de mortalidade para a pessoa idosa na UTI. Outros fatores importantes são citados, como o estado funcional prévio à admissão, presença de comorbidades, grau de severidade da doença, admissões não cirúrgicas, uso de ventilação mecânica e maior tempo de permanência até o desmame ventilatório. Todos esses fatores implicam em taxas de mortalidade elevadas para o grupo de pessoas idosas, tanto durante a internação em terapia intensiva quanto em até um ano após a alta.

Assim, é importante que a avaliação da pessoa idosa seja criteriosa e que a admissão em terapia intensiva seja discutida entre equipe multiprofissional, paciente e familiares, considerando o impacto de patologias subjacentes, comorbidades e gravidade da doença aguda na probabilidade de tratamento intensivo atingir o resultado desejado. Os riscos e os benefícios de qualquer tratamento intensivo e invasivo deve ser ponderado, contrapondo-os aos possíveis resultados prováveis.

\section{IMPLICAÇÕES DA COVID-19 NAS PESSOAS IDOSAS}

Um importante sistema do organismo humano, que deve estar a frente em infecções como a causada pelo novo coronavírus, é o sistema imunológico. No entanto, na pessoa idosa ocorre a diminuição da sua capacidade, em decorrência do processo natural do envelhecimento. Esse fenômeno denominado imunossenescência é traduzido por um conjunto de modificações, tanto na resposta inata quanto na adquirida. Tais modificações podem ocorrer em uma ou várias etapas do caminho de ativação celular, as quais resultarão em menor eficiência do sistema de defesa, aumentando a propensão e a gravidade de doenças infecciosas, autoimunes e neoplásicas ${ }^{(8)}$, fatores que podem explicar como a COVID-19 torna-se grave nestes indivíduos ${ }^{(10)}$. Dessa forma, pessoas idosas, especialmente aquelas com comorbidades associadas, apresentam maiores déficits no sistema imunológico, estando mais predispostos a mortalidade associadas à infecção viral.

Na população idosa algumas singularidades devem ser consideradas para a tomada de decisão diante da infecção pelo SARS-CoV-2, como os valores dos sinais vitais, dentre os quais, a temperatura. Tendo em vista que a hipertermia, como sintoma habitual em pacientes acometidos por qualquer quadro infeccioso, pode não ser um sinal suficientemente sensível em pessoas idosas, visto que essa frequentemente apresenta-se 
embotada ou ausente, mesmo em infecções graves ${ }^{(8)}$. Assim, a definição de hipertermia tem merecido algumas reflexões nesse contexto. Notabiliza-se que as alterações de temperatura podem ser ainda menos sensíveis entre as pessoas idosas mais frágeis.

Tendo em vista que as pessoas idosas tendem a apresentar temperaturas corporais menores do que as de adultos e jovens, pode-se seguir a recomendação de alerta, conforme indicações da Sociedade de Doenças Infecciosas da América, quando ocorrer a verificação de duas temperaturas repetidas acima de $37,2^{\circ} \mathrm{C}$ ou quando ocorrer um aumento de $\geq 1,3^{\circ} \mathrm{C}$ acima da temperatura basal. Outros critérios específicos que podem também indicar agravamento ou presença de um quadro infeccioso comumente encontrado nos idosos são síncope, confusão mental, sonolência excessiva, irritabilidade e inapetência.

Na prática clínica já são utilizados alguns pressupostos e protocolos clínicos que também podem ser úteis a esse contexto. Em relação aos exames laboratoriais, a proporção de pacientes com um número aumentado de glóbulos brancos e neutrófilos na população idosa, foi significativamente maior do que no grupo jovem e de meia idade, sugerindo que pessoas idosas infectados com a COVID-19, têm maior probabilidade de apresentar infecção bacteriana ${ }^{(10)}$.

Nessa perspectiva, reitera-se que as pessoas idosas são propensos à disfunção de órgãos multissistêmicos e até à falência, por isso outras complicações sistêmicas, que devem ser evitadas, incluindo sangramento gastrointestinal, insuficiência renal, Coagulação Intravascular Disseminada (CIVD) ou trombose venosa profunda ${ }^{(10)}$. Nesse contexto, quando há presença de infecções secundárias, uma abordagem multidisciplinar é recomendada e o ambiente de cuidados intensivos é o mais adequado.

Outros aspectos clínicos relevantes relacionados à COVID-19 merecem ser destacados, dentre os quais as manifestações nos exames de imagem, particularmente a Tomografia Computadorizada, que revela opacidades pulmonares em vidro fosco. A incidência de lesões multilobulares é significativamente maior em pessoas idosas do que em pacientes jovens e de meia idade ${ }^{(10)}$. No que se refere a duração média do início dos sintomas até a morte foi verificado que nas pessoas com mais de 70 anos esse período é de 11,5 dias e, em pessoas mais jovens de 14 dias $^{(10)}$.

Infere-se que esses resultados relacionados à gravidade da doença estejam relacionados às respostas imunes, que em pessoas idosas são mais lentas, menos coordenadas e menos eficientes, tornando-as mais suscetíveis à infecções emergentes. Ainda, com base no pressuposto de a função imune associa-se inversamente com a fragilidade e comorbidades, evidencia-se que uma pessoa idosa de 80 anos que é saudável e não é frágil pode ser mais resiliente no combate à infecção do que uma pessoa de 60 anos com muitas condições crônicas, pelo fato de ter um sistema imunológico mais jovem.

\section{CONSIDERAÇÕES FINAIS}

As pessoas idosas integram os grupos de risco para desenvolver desfechos desfavoráveis durante a pandemia do COVID-19, principalmente devido às características fisiológicas da senescência, bem como ao seu estado basal diante da senilidade. Diante do exposto, torna-se fundamental que a enfermagem de cuidados intensivos consiga identificar as alterações fisiológicas do ponto de vista físico, bem como as alterações cognitivas associadas ao envelhecimento, com vistas a garantir um cuidado diferenciado à pessoa idosa com COVID-19 em condições críticas ou potencialmente críticas de cuidados, com foco em prevenção de complicações, busca de resultados centrados no paciente e redução do tempo de internação hospitalar.

\section{AGRADECIMENTO}

Ao Departamento Científico de Enfermagem Gerontológica da ABEn Nacional. 


\section{REFERÊNCIAS}

1. World Health Organization (WHO). Q\&A on coronaviruses (COVID-19) [Internet]. [cited 2020 apr 18]. Available from: https://www.who.int/news-room/q-a-detail/q-a-coronaviruses

2. Pôrto, VA. Paciente idoso. In: Viana R, Torres M. Enfermagem em Terapia Intensiva: práticas integrativas. Barueri, SP: Manole, 2017.

3. Ministério da Saúde (Brasil). Boletim Epidemiológico Diário [Internet]. [cited 2020 apr 18]. Available from: https://www. saude.gov.br/images/pdf/2020/April/14/14.04.2020-COVID.pdf

4. Yang J, Zheng Y, Gou X, Pu K, Chen Z, Guo Q et al. Prevalence of comorbidities and its effects in coronavirus disease 2019 patients: A systematic review and meta-analysis. Int J Infect Dis [Internet]. 2020 [cited 2020 apr 18];94:91-95. Available from: https://doi.org/10.1016/j.ijid.2020.03.017

5. Wang D, Hu B, Hu C, Zhu F, Liu X, Zhang J et al. Clinical Characteristics of 138 Hospitalized Patients With 2019 Novel Coronavirus-Infected Pneumonia in Wuhan, China. JAMA [Internet]. 2020 [cited 2020 apr 22];323(11):1061-1069. Available from: 10.1001/jama.2020.1585

6. Lian J, JinX, Hao X, Cai H, Zhang S, Zheng L et al. Analysis of Epidemiological and Clinical features in older patients with Corona Virus Disease 2019 (COVID-19) out of Wuhan. Clin Infect Dis [Internet]. 2020 [cited 2020 apr 22];ciaa242. Available from: https://doi.org/10.1093/cid/ciaa242

7. Murthy S, Gomersall CD, Fowler RA. Care for Critically III Patients With COVID-19. JAMA [Internet]. 2020 [cited 2020 apr 22];323(15):1499-1500. Available from: 10.1001/jama.2020.3633

8. Freitas EV, Py L. Tratado de geriatria e gerontologia. 4. ed. Rio de Janeiro: Guanabara Koogan, 2016.

9. Guimarães HP, Schubert DUC, Rodrigues RR, Freitas APR, Corrêa TD, Cunha KA et al. Recomendações para Intubação Orotraqueal em pacientes portadores de COVID-19 Versão N.3/2020. Associação Brasileira de Medicina de Emergência (ABRAMED) e Associação de Medicina Intensiva Brasileira (AMIB) [Internet]. 2020 [cited 2020 apr 22]. Available from: http://abramede.com.br/wp-content/uploads/2020/04/Recomendacoes-IOT-FINAL-REVISAO-100420.pdf

10. Liu K, Chen Y, Lin R, Han K. Clinical features of COVID-19 in elderly patients: A comparison with young and middle-aged patients. J Infect [Internet]. 2020 [cited 2020 apr 22]. Available from: doi:10.1016/j.jinf.2020.03.005 